\title{
Design and Realization of a New Signal Security System for Multimedia Data Transmission
}

\author{
Hun-Chen Chen \\ Department of Electronics Engineering, National United University, Miaoli 360, Taiwan
}

Email:hcchen@nuu.edu.tw

\section{Jiun-In Guo}

Department of Computer Science and Information Engineering, National Chung Cheng University, Chia-Yi 621, Taiwan Email: jiguo@cs.ccu.edu.tw

\author{
Lin-Chieh Huang \\ Department of Computer Science and Information Engineering, National Chung Cheng University, Chia-Yi 621, Taiwan \\ Email:hlc91@cs.ccu.edu.tw
}

\author{
Jui-Cheng Yen \\ Department of Electronics Engineering, National United University, Miaoli 360, Taiwan \\ Email: jcyen@nuu.edu.tw
}

Received 22 January 2003 and in revised form 25 August 2003

\begin{abstract}
We propose a new signal security system and its VLSI architecture for real-time multimedia data transmission applications. We first define two bit-circulation functions for one-dimensional binary array transformation. Then, we exploit a chaotic system in generating a binary sequence to control the bit-circulation functions defined for performing the successive transformation on the input data. Each eight 8-bit data elements is regarded as a set and is fed into an $8 \times 8$ binary matrix being transformed on each row and each column of the matrix by these two bit-circulation functions such that the signal can be transformed into completely disordered data. The features of the proposed design include low computational complexity, regular operations suitable for lowcost VLSI implementation, high data security, and high feasibility for easy integration with commercial multimedia storage and transmission applications. We have performed Matlab simulation to verify the functional correctness of the proposed system. In implementing the system, a low-cost VLSI architecture has been designed, verified, and physically realized based on a $0.35 \mu \mathrm{m}$ CMOS technology. The implementation results show that the proposed signal security system can achieve $117 \mathrm{Mbytes} / \mathrm{s}$ data throughput rate that is fast enough for real-time data protection in multimedia transmission applications.
\end{abstract}

Keywords and phrases: signal encryption/decryption, VLSI chip, chaotic system, and fractal dimension.

\section{INTRODUCTION}

Recently, with the great demand in digital signal transmission $[1,2]$ and the big losses from illegal data access, data security has become a critical and imperative issue in the multimedia data transmission applications. In order to protect valuable data from undesirable readers or against illegal reproduction and modifications, there have been various data encryption techniques $[3,4,5,6,7,8,9,10]$ and the watermark embedding schemes $[11,12,13]$ on images proposed in the literature. The data encryption techniques make the images invisible to undesirable readers and can be applied to protect the frames in the digital versatile disk (DVD) and the cable TV, while the watermark-embedded schemes hide the watermark onto an image to declare their ownership but the image is still visible.

Among the existing data encryption techniques $[3,4,5$, $6,7,8,9,10]$, we can classify their basic design ideas into three major types: position permutation $[5,6]$, value transformation $[7,8]$, and the combination form $[9,10]$. The position permutation algorithms scramble the original data according to some predefined schemes. It is simple but usually has low data security. The value transformation algorithms transform the data value of the original signal with some kinds of transformation. It has the potential of low computational complexity and low hardware cost. Finally, the combination form performs both position permutation and value transformation. It has the potential of high data security. 


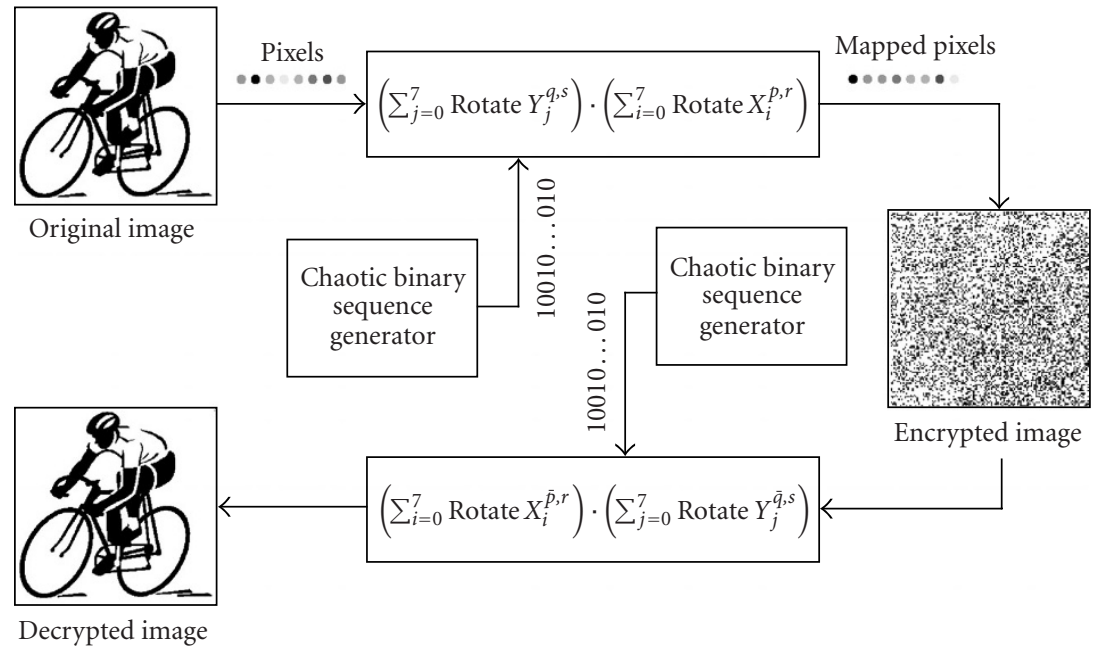

FIGURE 1: The block diagram of the proposed signal security system applied to a still image encryption/decryption.

In this paper, we propose a new signal security system and its VLSI architecture for real-time multimedia data transmission applications. The proposed encryption algorithm belongs to the category of the combination form mentioned above. We first define two bit-circulation functions with two parameters in each function. One is used to control the shift direction and the other is used to control the shifted bit-number on the data transformation. Then, we exploit a chaotic system in generating a binary sequence to control the bit-circulation functions for performing the successive data transformation on the input data. Eight 8-bit data elements are regarded as a set and fed into an $8 \times 8$ binary matrix. In the successive transformation on each row and each column by using these two functions, we randomly determine the two parameters used in the functions according to the generated chaotic binary sequence such that the signal can be transformed into completely disorderly data. In demonstrating the correct functionality of the proposed signal security system, we have performed the Matlab simulation on the proposed scheme. In implementing the proposed system, we present a low-cost VLSI architecture that has been designed, verified, and physically realized by using Verilog hardware description language (HDL), Synopsys logic synthesis tool design compiler (DC), and Avanti layout tools (Apollo) based on a $0.35 \mu \mathrm{m}$ CMOS technology. The implementation results show that the proposed signal security system can achieve $117 \mathrm{Mbytes} / \mathrm{s}$ data throughput rate at the cost of silicon area of $3.59 \mathrm{~mm}^{2}$. This data-processing rate is fast enough for realtime data protection in multimedia data transmission applications.

The proposed signal security system is suitable for both software and hardware implementation depending on the requirement of applications. In the multimedia applications realized in software or DSP firmware, it is suggested to realize the proposed system through general-purpose processors or DSP processors. On the other hand, it is suggested to use the proposed hardware design in the multimedia applications realized in hardware like ASICs or SOCs. In this situation, the system integrators can use the proposed encryption/decryption design as an independent module or intellectual properties (IP) that can be cooperated with the existing multimedia ASICs or SOCs to perform the functionality of real-time data encryption and decryption.

The rest of this paper is organized as follows. In Section 2, we propose the new signal security system including algorithm derivation and illustration as well as the analysis on complexity and security. In Section 3, we perform the software simulation, randomness measurement, and sensitivity analysis of parameters in the proposed system. In Section 4, we illustrate the hardware design and realization of the proposed system. In Section 5, we evaluate the performance evaluation of the proposed design and compare it with the existing designs. Finally, we conclude this paper in Section 6.

\section{THE PROPOSED NEW SIGNAL SECURITY SYSTEM}

\subsection{Notations and definitions}

Let $g$ denote a one-dimensional (1D) digital signal of length $N, g(n), 0 \leq n \leq N-1$, be the one-byte value of the signal $g$ at $n, M$ an $8 \times 8$ binary matrix, and $g^{\prime}$ and $M^{\prime}$ the encryption results of $g$ and $M$, respectively. In the following definitions, the integer parameters $r$ and $s$ are assumed to be larger than or equal to 0 , but they are less than 8 .

Definition 1. The mapping Rotate $X_{i}^{p, r}: M \rightarrow M^{\prime}$ is defined to rotate each bit in the $i$ th row of $M, 0 \leq i \leq 7, r$ bits in the left direction if $p$ equals 1 or $r$ bits in the right direction if $p$ equals 0 .

Definition 2. The mapping Rotate $Y_{j}^{q, s}: M \rightarrow M^{\prime}$ is defined to rotate each bit in the $j$ th column of $M, 0 \leq j \leq 7, s$ bits in the up direction if $q$ equals 1 or $s$ bits in the down direction if $q$ equals 0 . 
For example, let

$$
M=\left[\begin{array}{llllllll}
1 & 0 & 0 & 0 & 0 & 1 & 1 & 1 \\
1 & 1 & 0 & 0 & 1 & 0 & 1 & 1 \\
1 & 0 & 1 & 0 & 0 & 1 & 1 & 1 \\
1 & 1 & 1 & 1 & 0 & 0 & 0 & 1 \\
0 & 1 & 1 & 0 & 0 & 0 & 0 & 0 \\
1 & 1 & 1 & 0 & 0 & 1 & 1 & 1 \\
0 & 0 & 1 & 0 & 1 & 0 & 0 & 0 \\
0 & 0 & 1 & 1 & 1 & 0 & 0 & 0
\end{array}\right],
$$

then,

$$
\begin{aligned}
& \text { Rotate } X_{3}^{1,3}(M)=\left[\begin{array}{llllllll}
1 & 0 & 0 & 0 & 0 & 1 & 1 & 1 \\
1 & 1 & 0 & 0 & 1 & 0 & 1 & 1 \\
1 & 0 & 1 & 0 & 0 & 1 & 1 & 1 \\
1 & 0 & 0 & 0 & 1 & 1 & 1 & 1 \\
0 & 1 & 1 & 0 & 0 & 0 & 0 & 0 \\
1 & 1 & 1 & 0 & 0 & 1 & 1 & 1 \\
0 & 0 & 1 & 0 & 1 & 0 & 0 & 0 \\
0 & 0 & 1 & 1 & 1 & 0 & 0 & 0
\end{array}\right] \\
& \text { Rotate } Y_{3}^{1,3}(M)=\left[\begin{array}{llllllll}
1 & 0 & 0 & 1 & 0 & 1 & 1 & 1 \\
1 & 1 & 0 & 0 & 1 & 0 & 1 & 1 \\
1 & 0 & 1 & 0 & 0 & 1 & 1 & 1 \\
1 & 1 & 1 & 0 & 0 & 0 & 0 & 1 \\
0 & 1 & 1 & 1 & 0 & 0 & 0 & 0 \\
1 & 1 & 1 & 0 & 0 & 1 & 1 & 1 \\
0 & 0 & 1 & 0 & 1 & 0 & 0 & 0 \\
0 & 0 & 1 & 0 & 1 & 0 & 0 & 0
\end{array}\right] \text {, } \\
& \text { Rotate } Y_{5}^{0,2} \cdot \operatorname{Rotate} X_{2}^{1,2}(M)=\left[\begin{array}{llllllll}
1 & 0 & 0 & 0 & 0 & 0 & 1 & 1 \\
1 & 1 & 0 & 0 & 1 & 0 & 1 & 1 \\
1 & 0 & 0 & 1 & 1 & 1 & 1 & 0 \\
1 & 1 & 1 & 1 & 0 & 0 & 0 & 1 \\
0 & 1 & 1 & 0 & 0 & 1 & 0 & 0 \\
1 & 1 & 1 & 0 & 0 & 0 & 1 & 1 \\
0 & 0 & 1 & 0 & 1 & 0 & 0 & 0 \\
0 & 0 & 1 & 1 & 1 & 1 & 0 & 0
\end{array}\right] \text {. }
\end{aligned}
$$

In different combinations of $p, q, r$, and $s$, the composite mapping $\left(\sum_{j=0}^{7}\right.$ Rotate $\left.Y_{j}^{q, s}\right) \cdot\left(\sum_{i=0}^{7}\right.$ Rotate $\left.X_{i}^{p, r}\right)$ possesses the following three desirable features:

(1) a binary matrix $M$ can be transformed into quite different matrixes;

(2) different matrixes can be transformed into the same matrix;

(3) given a transformation pair of $M$ and $M^{\prime}$, the combination of $p, q, r$, and $s$ resulting in the transformation pair may be nonunique.

Since $M$ is an $8 \times 8$ matrix, the result of circulating its row or column $k$ bits is equal to the result of circulating it $(k \bmod 8)$ bits in the same direction. This is why $r$ and $s$ are assumed to be in the ranges of $0 \leq r \leq 7$ and $0 \leq s \leq 7$.

\subsection{The new signal security system}

Based on the notations and definitions, the encryption procedure denoted as the two-dimensional (2D) circulation encryption algorithm (TDCEA) on $g$ is proposed in Algorithm 1.

Each eight 8-bit data elements are regarded as a processing group and fed into the $8 \times 8$ binary matrix $M$. Each row of $M$ is transformed by Rotate $X_{i}^{p, r}$, and then each column of the resulting matrix is transformed by Rotate $Y_{j}^{q, s}$. In each row or column transformation, the mapping parameters $p, r, q$, and $s$ are randomly determined by the chaotic binary sequence in (3), (4), (6), and (7). The row transformation belongs to the type of value transformation and the column transformation makes the position permutation in each bit-plane. Hence, the TDCEA belongs to the type of combination-form category in the existing data encryption schemes.

The decryption procedure is very similar to Algorithm 1 except for the following two modifications.

(1) The $j$-loop including the assignment of $q$ and $s$ and the mapping Rotate $Y_{j}^{q, s}$ is changed to be ahead of the $i$ loop including the assignment of $p$ and $r$ and the mapping Rotate $X_{i}^{p, r}$.

(2) The parameter $q$ in Rotate $Y_{j}^{q, s}$ changes to its complement $\operatorname{INV}(q)$ and the parameter $p$ in Rotate $X_{i}^{p, r}$ changes to its complement $\operatorname{INV}(p)$, where $\operatorname{INV}(x)$ denotes the logically inverting operation on the variable $x$, that is, the mapping function applied to $M^{\prime}$ in the decryption subsystem becomes $\left(\sum_{i=0}^{7}\right.$ Rotate $\left.X_{i}^{\bar{p}, r}\right)$. $\left(\sum_{j=0}^{7}\right.$ Rotate $\left.Y_{j}^{\bar{q}, s}\right)$.

Combining the encryption subsystem and decryption subsystem, the block diagram of the proposed signal security system is shown in Figure 1. By extracting 17 bits from each evolution state of the logistic map, we generate a binary sequence. The reason why we adopt 17 bits depends on the amount of control signals needed in each cycle when applying the proposed TDCEA.

Then, the sequence is used to control the parameters in $\left(\sum_{j=0}^{7}\right.$ Rotate $\left.Y_{j}^{q, s}\right) \cdot\left(\sum_{i=0}^{7} \operatorname{Rotate} X_{i}^{p, r}\right)$ according to (3), (4), (6), and (7) in Algorithm 1. That is, the rotation direction and the shifted bit-number in the mapping are randomly controlled by the sequence. Finally, each eight pixels are successively mapped and the completely chaotic results can be obtained. In the decryption phase, according to the same $\mu$ and $x(0)$, that is, the same chaotic binary sequence, the original image can be correctly reconstructed by applying $\left(\sum_{i=0}^{7}\right.$ Rotate $\left.X_{i}^{\bar{p}, r}\right) \cdot\left(\sum_{j=0}^{7}\right.$ Rotate $\left.Y_{j}^{\bar{q}, s}\right)$ to the encrypted image. The variables $\mu$ and $x(0)$ used in the proposed algorithm can be protected and transmitted from transmitters to receivers using the method illustrated in Section 2.3.

\subsection{Generation, protection, and transmission of the parameters $\alpha, \beta, \mu$, and $x(0)$}

In the proposed design, we need four parameters $\alpha, \beta, \mu$, and $x(0)$ in generating the chaotic bit-stream from the $1 \mathrm{D}$ logistic 
Step 1: Determine the parameters $N, \alpha$, and $\beta$, where $0<\alpha+\beta<8, \alpha \in \mathbb{N}$, and $\beta \in(\mathbb{N} \cup\{0\})$, where $\mathbb{N}$ denotes the set of positive integers.

Step 2: Determine the parameter $\mu$ and the initial point $x(0)$ of the 1D logistic map $f_{\mu}(x)=\mu x(1-x)$. Evolve successive states from the $1 \mathrm{D}$ logistic map [14,15] by $x(n+1)=\mu x(n)(1-x(n))$, and the preceding 17 bits below the decimal point of the binary representation of $x(n), n=1,2, \ldots$, are extracted to constitute the chaotic binary sequence $b(0), b(1), b(2), \ldots$, and so forth.

Step 3: For $k=0$ to $(N / 8-1)$ Do

$$
\begin{aligned}
& \text { For } x=0 \text { to } 7 \mathrm{Do} \\
& \text { Let } g(8 k+x)=\sum_{y=0}^{7} d_{y} \times 2^{y} \text {; } \\
& \text { For } y=0 \text { to } 7 \\
& \text { End } \\
& M(x, y)=d_{y} ; \\
& M=\text { Rotate } X_{i}^{p, r}(M) \text {; }
\end{aligned}
$$

End

For $j=0$ to 7 Do

$$
\begin{aligned}
q & =b(17 k+8+j), \\
s & =\alpha+\beta \times b(17 k+9+j), \\
M & =\text { Rotate } Y_{j}^{q, s}(M)
\end{aligned}
$$

End

For $x=0$ to 7 Do

$$
g^{\prime}(8 k+x)=\sum_{y=0}^{7} M(x, y) \times 2^{y}
$$

End

End

Step 4: The encryption result $g^{\prime}$ is obtained and the algorithm is terminated.

Algorithm 1: The two-dimensional circulation encryption algorithm (TDCEA).

map. The four parameters could be viewed as the keys to the proposed signal security system. Among them, the parameters $\alpha$ and $\beta$ can be fixed in both the transmitter and receiver according to the constraint shown in Step 1 of the proposed TDCEA. In providing higher security of the proposed TDCEA, we can try to vary the parameters of $\mu$ and $x(0)$ during the transmission of multimedia data frequently. Let $\mu_{b}$ and $X_{b}$ denote the built-in keys of $\mu$ and $x(0)$, respectively, in the proposed signal security system. Let $\mu_{\mathrm{enc}, 0}$ and $X_{\mathrm{enc}, 0}$ denote the initial encrypting keys of $\mu$ and $x(0)$, respectively, during the transmission from the transmitter to the receiver. The way of generating, protecting, and transmitting the parameters of $\mu$ and $x(0)$ is shown in the following procedures.

(1) We first select the initial values of $\mu_{0}$ and $x(0)_{0}$ following the constraints of $3<\mu_{0}<4$ and $0<x(0)_{0}<1$.

(2) Then, we encrypt the initial $\mu_{0}$ and $x(0)_{0}$ by the following way:

$$
\begin{aligned}
& X_{\text {enc }, 0}=x(0)_{0} \oplus X_{b}, \\
& \mu_{\text {enc }, 0}=\mu_{0} \oplus \mu_{b} .
\end{aligned}
$$

(3) Transmitting the encrypted $\mu_{\mathrm{enc}}$ and $X_{\mathrm{enc}}$ from the transmitter to the receiver for reproducing the initial $\mu_{0}$ and $x(0)_{0}$ by the following way:

$$
\begin{aligned}
x(0)_{0} & =X_{\text {enc }, 0} \oplus X_{b}, \\
\mu_{0} & =\mu_{\text {enc }, 0} \oplus \mu_{b} .
\end{aligned}
$$

(4) In the $p$ th updating of the parameters $\mu$ and $x(0)$, that is, $\mu_{p}$ and $x(0)_{p}$, we can use the values of $\mu_{p-1}$ and $x(0)_{p-1}$ in the $(p-1)$ th updating to replace the roles of the built-in parameters of $\mu_{b}$ and $X_{b}$. Then, we can use the way specified in (10) and (11) in protecting the successive updated parameters of $\mu$ and $x(0)$ as follows:

$$
\begin{aligned}
X_{\text {enc }, p} & =x(0)_{p} \oplus x(0)_{p-1}, \\
\mu_{\text {enc }, p} & =\mu_{p} \oplus \mu_{p-1}, \\
x(0)_{p} & =X_{\text {enc, } p} \oplus x(0)_{p-1}, \\
\mu_{p} & =\mu_{\text {enc }, p} \oplus \mu_{p-1} .
\end{aligned}
$$

(5) The updating period of the parameters of $\mu$ and $x(0)$ could be based on the basic unit of video frame or audio frame in representing the multimedia data. 
TABLE 1: The complexity of the proposed TDCEA on a signal of length $N$.

\begin{tabular}{|c|c|c|c|c|}
\hline Operation & Multiplication & Addition/subtraction & Condition test & $\ll$ or $\gg$ \\
\hline Step 2 & $2\lfloor N / 8\rfloor$ & $\lfloor N / 8\rfloor$ & 0 & 0 \\
\hline Equation (3) of Step 3 & $N$ & $N$ & 0 & 0 \\
\hline Equation (4) of Step 3 & $N$ & $2 N$ & $N$ & 0 \\
\hline Equation (5) of Step 3 & 0 & 0 & 0 & $N$ \\
\hline Equation (6) of Step 3 & $N$ & $N$ & 0 & 0 \\
\hline Equation (7) of Step 3 & $N$ & $2 N$ & $N$ & 0 \\
\hline Equation (8) of Step 3 & 0 & 0 & 0 & $N$ \\
\hline Total & $4 N+2\lfloor N / 8\rfloor$ & $6 N+\lfloor N / 8\rfloor$ & $2 N$ & $2 N$ \\
\hline
\end{tabular}

\subsection{Chaos via 1D logistic map}

A simple and well-studied example of a 1D map [14] that exhibits complicated behavior is the logistic map from the interval $[0,1]$ into $[0,1]$, parameterized by $\mu$ :

$$
g_{\mu}(x)=\mu x(1-x)
$$

where $0 \leq \mu \leq 4$. This map constitutes a discrete-time dynamical system in the sense that the map $g_{\mu}:[0,1] \rightarrow[0,1]$ generates a semigroup through the operation of composition of functions. The state evolution is described by $x_{n+1}=$ $g_{\mu}\left(x_{n}\right)$. We denote

$$
g^{(n)} \equiv g \circ g \circ \cdots \circ g \quad(n \text { times }) .
$$

For all $x \in[0,1]$, a "discrete-time" trajectory $\left\{x_{i}\right\}_{i=0}^{\infty}$, where $x_{i}=g^{(i)}(x)$, can be generated. The set of points $\left\{x_{0}, x_{1}, \ldots\right\} \subset$ $[0,1]$ is called the (forward) orbit of $x$. A periodic point of $g$ is a point $x \in[0,1]$ such that $x=g^{(n)}(x)$ for some positive integer $n$. The least positive integer $n$ is called the period of $x$. A periodic point of period 1 is called a fixed point. For differentiable $g$, a periodic point $x$ with period $n$ is stable if

$$
\left|\prod_{i=1}^{n} g^{\prime}\left(x_{i}\right)\right|<1
$$

and unstable if

$$
\left|\prod_{i=1}^{n} g^{\prime}\left(x_{i}\right)\right|>1
$$

where $x_{i}=g^{(i)}(x)$.

In the logistic map, as $\mu$ is varied from 0 to 4 , a perioddoubling bifurcation occurs. In the region $\mu \in[0,3]$, the map $g_{\mu}$ possesses one stable fixed point. As $\mu$ is increased past 3 , the stable fixed point becomes unstable and two new stable periodic points of period 2 are created. As $\mu$ is further increased, these stable periodic points in turn become unstable and each spawns two new stable periodic points of period 4. Thus the period of the stable periodic points is doubled at each bifurcation point. Each period-doubling episode occurs in a shorter "parameter" interval, decreasing at a geometric rate each time. Moreover, at a finite $\mu$, the period-doubling

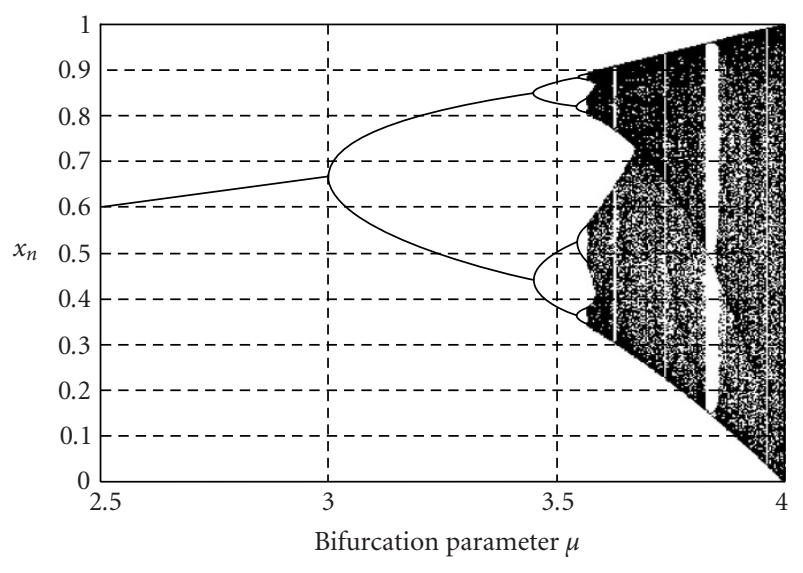

Figure 2: Bifurcation diagram of the logistic map $g_{\mu}(x)=\mu x(1-x)$.

episode converges to an infinite number of period doublings at which point chaos is observed. This is depicted in the bifurcation diagram in Figure 2.

\subsection{Analysis of computational complexity}

If the TDCEA is simulated by using $C$ language, just the basic bitwise operators " $\ll$ " and " $\gg$ " can accomplish the mappings Rotate $X_{i}^{p, r}$ and Rotate $Y_{j}^{q, s}$. In Step 2, it requires one subtraction and two multiplications to evolve a state from the 1D logistic map, and the total evolution number is $\lfloor N / 8\rfloor$, where $\lfloor y\rfloor$ denotes the largest integer that is smaller than or equal to $y$. In (4) and (7), since $b(n)$ is either 1 or 0 , the equations can be performed by one condition test and two additions instead. The computational complexity of the proposed TDCEA on a signal of length $N$ is listed in Table 1. From Table 1, the numbers of multiplications, additions/subtractions, condition tests, and circular shiftings are $4 N+2\lfloor N / 8\rfloor, 6 N+\lfloor N / 8\rfloor, 2 N$, and $2 N$, respectively. Hence, the computational complexity of the TDCEA is proportional to $O(N)$.

\subsection{Analysis of security problem}

It is of interests to know if the TDCEA is easily decrypted or not. This security problem is analyzed in the following. 
Proposition 1. For an unknown set of $\mu$ and $x(0)$ of the logistic map, the number of possible encryption results is $2^{17 N / 8}$ if the TDCEA is applied to a signal of length $N$.

Proof. Since it requires $17 N / 8$ bits to encrypt a signal of length $N$, the number of possible encryption results is $2^{17 N / 8}$.

For example, consider an image of size $256 \times 256$ pixels. In this case, $N$ equals 65536 . All the possibilities are $2^{139264}\left(\cong 10^{41918}\right)$. Since the chaotic binary sequence is unpredictable [16], it is very difficult to decrypt correctly an encrypted signal by making an exhaustive search without knowing $\mu$ and $x(0)$. Moreover, small fluctuation in $\mu$ and $x(0)$ results in quite different chaotic binary sequence because the trajectory of the chaotic system is very sensitive to initial condition [16]. By way of collecting some original signals and their encryption results or collecting some specified signals and their corresponding encryption results, it is impossible for the cryptanalysts to decrypt correctly an encrypted image without knowing $\mu$ and $x(0)$ because the rotation direction and the shifted bit-number in each row or column transformation is randomly determined by the chaotic binary sequence. Hence, the new scheme can resist the chosen ciphertext attack and the known plaintext attack [17].

\section{SIMULATION RESULTS}

\subsection{Software simulation and the calculation of fractal dimension}

In the simulation, ten images of size $256 \times 256$ are used. As representatives, only the images of "cman," "aero," and "pepper" are shown in Figures 3a, 3d, and 3g, respectively. The most direct method to decide the disorderly degree of the encrypted image is by the sense of sight. On the other hand, the fractal dimension $[18,19]$ can provide the quantitative measure on the randomness of the encrypted images. General images typically have a degree of randomness associated with both the natural random nature of the underlying structure and the random noise superimposed on the image. An image $f$ of size $L \times P$ pixels is regarded as a surface with $z=f(x, y)$ in $\mathbb{R}^{3}$. To measure how rough the encrypted image surface is, its fractal dimension $D$ is calculated according to the method in [19].

Let ndi $(k)$ be the average of absolute intensity difference of all pixel pairs with distance values whose integer parts are $k$. The value of $\operatorname{ndi}(k)$ is computed by

$$
\begin{aligned}
& \operatorname{ndi}(k) \\
& =\frac{\sum_{x 1=0}^{L-1} \sum_{y 1=0}^{P-1} \sum_{x 2=0}^{L-1} \sum_{y 2=0}^{P-1}|f(x 2, y 2)-f(x 1, y 1)|}{\operatorname{npn}(k)},
\end{aligned}
$$

where $\operatorname{npn}(k)$ is the total number of pixel pairs with distance $\Delta r$ such that $k \leq \Delta r<k+1$, and $x 1, y 1, x 2$, and $y 2$ must satisfy

$$
k \leq \sqrt{(x 2-x 1)^{2}+(y 2-y 1)^{2}}<k+1 .
$$

Plot all pairs $(\log (k), \log (\operatorname{ndi}(k)))$, and then use a leastsquares linear regression to estimate the slope $H$ of the resultant curve. The fractal dimension $D=3-H$ can be obtained. In the simulation, the maximal distance $k$ between two pixels in (17) is set to 60 .

In order to apply the TDCEA, the parameters $\alpha$ and $\beta$ must be determined according to Step 1. Basically, the selection of $\alpha$ and $\beta$ should follow the empirical law. According to the experimental experience, general combinations of $\alpha$ and $\beta$ can always result in very disorderly results. In the simulation, $\alpha=2$ and $\beta=2$ are adopted in Step 1. In the logistic map, $x(0)=0.75$ and $\mu=3.9$ are set in Step 2. The encrypted results of the three representative images by the TDCEA are shown in Figures 3b, 3e, and 3h. Moreover, the fractal dimensions of the original images and their encryption results are calculated and listed in Table 2.

According to Figure 3, the encryption results of the TDCEA are of complete disorder and cannot be distinguished from the original ones. Moreover, from the quantitative measure results shown in Table 2, the fractal dimensions of the encrypted images range from 2.9974 to 2.9830 . Since the maximal fractal dimension for a $2 \mathrm{D}$ surface is 3.00 , the encryption results of the TDCEA are completely disordered. Figures $3 \mathrm{c}, 3 \mathrm{f}$, and $3 \mathrm{i}$, respectively, show the decrypted images of "cman," "aero," and "pepper." Since the proposed TDCEA is not losable, we can find that there would be no encryption/decryption errors in using the proposed TDCEA.

\subsection{Analysis of parameter sensitivity}

In order to demonstrate that the encryption results of the TDCEA are very sensitive to $\mu$ and $x(0)$, tiny fluctuation in the two parameters is considered. To compare the encryption results with small parameter fluctuation, the root mean square difference (RMSD) is computed. Let $f_{\mu_{1}, x_{1}(0)}^{\prime}$ be the encryption result of the image $f$ under $\mu_{1}$ and $x_{1}(0)$ and let $f_{\mu_{2}, x_{2}(0)}^{\prime}$ be the one under $\mu_{2}$ and $x_{2}(0)$; the RMSD between $f_{\mu_{1}, x_{1}(0)}^{\prime}$ and $f_{\mu_{2}, x_{2}(0)}^{\prime}$ is defined as

$\operatorname{RMSD} \equiv\left(\frac{1}{L \times P} \sum_{i=0}^{L-1} \sum_{j=0}^{P-1}\left(f_{\mu_{1}, x_{1}(0)}^{\prime}(i, j)-f_{\mu_{2}, x_{2}(0)}^{\prime}(i, j)\right)^{2}\right)^{1 / 2}$,

where $f$ is an image of size $L \times P$ pixels. Firstly, $x(0)$ is fixed to be 0.75 and tiny fluctuation of $10^{-5}$ in $\mu$ is considered. The RMSD comparison result of "Lena" is listed in Table 3. Secondly, $\mu$ is fixed to be 3.9 and tiny fluctuation of $10^{-5}$ in $x(0)$ is considered. The RMSD between each fluctuation is listed in Table 4. From Tables 3 and 4, the RMSDs range from 91.1739 to 92.6910 . The average gray-level difference of each pixel between the two encryption results with tiny fluctuation in $\mu$ or $x(0)$ is about 92 . It implies that the two results are extraordinarily different. Hence, the encryption result of the TDCEA is very sensitive to the fluctuation in $\mu$ and $x(0)$.

\section{ARCHITECTURE DESIGN AND REALIZATION}

Figure 4a shows the architecture of the proposed new signal security system. It includes one signal encryption unit (SEU), 


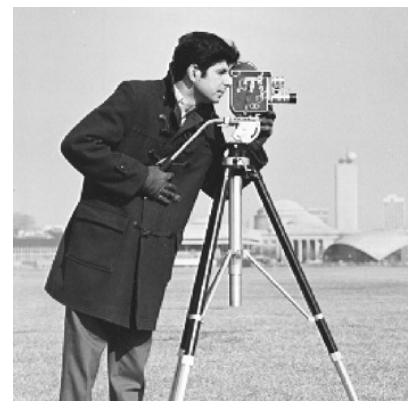

(a)

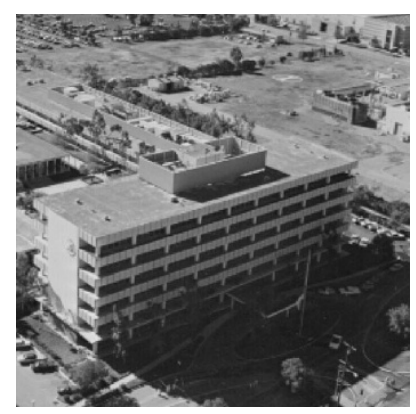

(d)

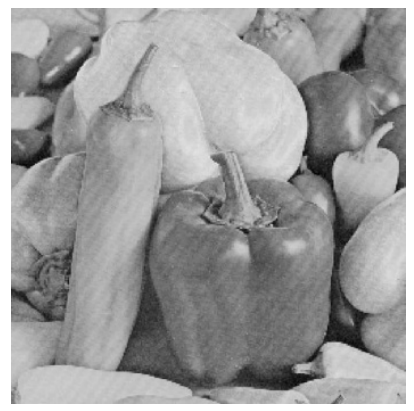

(g)

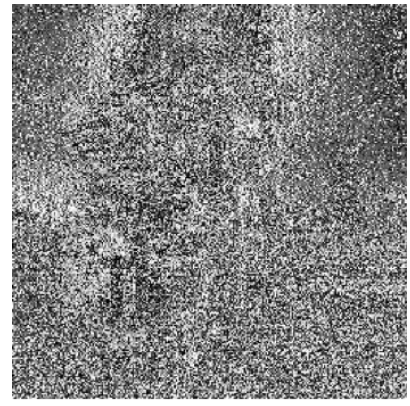

(b)

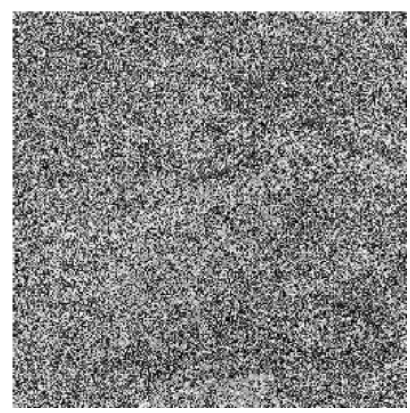

(e)

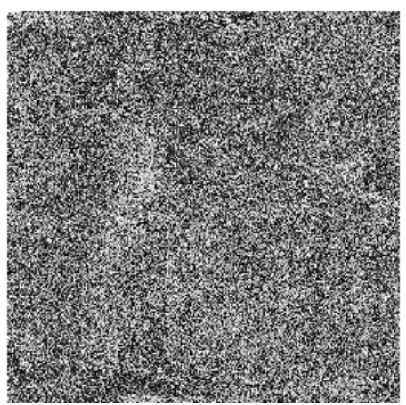

(h)

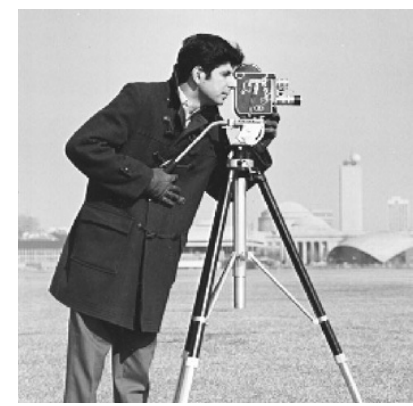

(c)

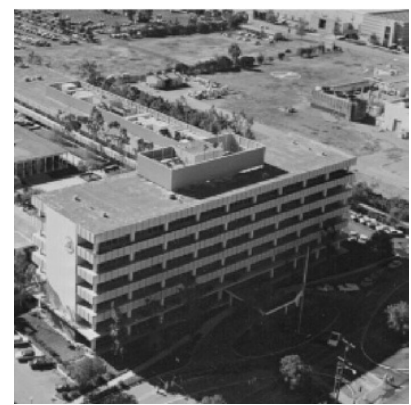

(f)

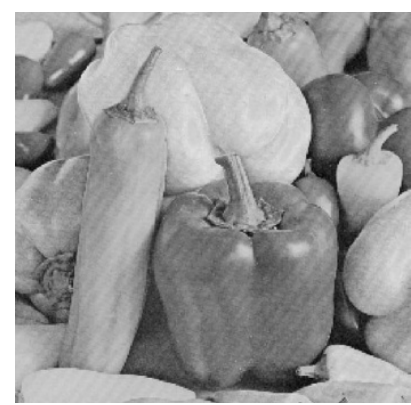

(i)

Figure 3: (a) Original "Cman," (b) encrypted "Cman," (c) decrypted "Cman," (d) original "Aero," (e) encrypted "Aero," (f) decrypted "Aero," (g) original "Pepper," (h) encrypted "Pepper," and (i) decrypted "Pepper."

TABLE 2: The fractal dimensions of the original and encrypted images.

\begin{tabular}{l|cccccccccc}
\hline & Cman & Einstein & Mill & Lena & Aero & Baboon & Pepper & Boat & Boys & Karen \\
\hline Original Image & 2.5671 & 2.6702 & 2.8087 & 2.5993 & 2.7427 & 2.7178 & 2.6407 & 2.7885 & 2.4878 & 2.5932 \\
Encrypted Image & 2.9830 & 2.9937 & 2.9957 & 2.9924 & 2.9957 & 2.9974 & 2.9947 & 2.9894 & 2.9906 & 2.9901 \\
\hline
\end{tabular}

TABLE 3: The RMSD between the encryption results with $x(0)=0.75$ and tiny fluctuation of $10^{-5}$ in $\mu$.

\begin{tabular}{c|ccccccccc}
\hline \multirow{4}{*}{$\mu$} & 3.9000 & 3.90001 & 3.90002 & 3.90003 & 3.90004 & 3.90005 & 3.90006 & 3.90007 & 3.90008 \\
vs. & vs. & vs. & vs. & vs. & vs. & vs. & vs. & vs. & 3.900 \\
& 3.90001 & 3.90002 & 3.90003 & 3.90004 & 3.90005 & 3.90006 & 3.90007 & 3.90008 & 3.90009 \\
\hline RMSD & 91.7405 & 91.7855 & 92.0908 & 91.7223 & 92.4095 & 92.0035 & 92.0625 & 92.1774 & 91.9920 \\
\hline
\end{tabular}


TABLE 4: The RMSD between the encryption results with $\mu=3.9$ and tiny fluctuation of $10^{-5}$ in $x(0)$.

\begin{tabular}{c|ccccccccc}
\hline \multirow{3}{*}{$x(0)$} & 0.75000 & 0.75001 & 0.75002 & 0.75003 & 0.75004 & 0.75005 & 0.75006 & 0.75007 & 0.75008 \\
& vs. & vs. & vs. & vs. & vs. & vs. & vs. & vs. & vs. \\
& 0.75001 & 0.75002 & 0.75003 & 0.75004 & 0.75005 & 0.75006 & 0.75007 & 0.75008 & 0.75009 \\
\hline RMSD & 92.0361 & 92.0357 & 92.2701 & 92.5990 & 92.6580 & 92.1739 & 91.8318 & 92.4014 & 92.6910 \\
\hline
\end{tabular}

one signal decryption unit (SDU), and two chaotic binary sequence generators (CBSGs). From the proposed algorithm, we find that the SDU operation is very similar to the SEU operation except that the directions of the left/right shifting on the row data and the up/down shifting on the column data are opposite. So, with opposite control signals in the shifting directions, the same SEU architecture can be used in the SDU. Thus, we design an encryption/decryption core for the proposed system as shown in Figure $4 \mathrm{~b}$. Figure 5 shows the architecture of the SEU/SDU in the encryption/decryption core. In the SEU/SDU design, we adopt the 2D array of processing elements (PEs) as shown in Figure 6 to perform the signal encryption/decryption operation on eight pixels simultaneously in bit level. That is, each PE has been designed to perform the following functions including loading, left/right shifting, and up/down shifting under the control of the parameters $p, q, r$, and $s$, derived from the chaotic binary sequence $b(\cdot)$ that is obtained from the CBSG as shown in Figure 7. As shown in Figure 6, the PE in the proposed design is composed of the data multiplexing circuit and D-type flipflop (DFF). Using data multiplexing circuit is to achieve the loading/outputting, left/right (L/R) rotation, and up/down (U/D) rotation using the same DFF. Since the shifting operations with variant numbers of bits are needed in the proposed design, we use DFF with configurable input sources to achieve this goal in multiple clock cycles instead of disabling the clock signal to the DFF. For each left/right shifting on the row data, it takes 8 cycles to finish the operations in the worst case. Similarly, it also takes 8 cycles to finish the up/down shifting operations for the column data in the worst case. Including the initial 8 cycles needed to feed the input data into the 2D array of PEs, it totally takes 24 cycles to finish the signal encryption operation for each 8 input pixels. For balancing the $\mathrm{I} / \mathrm{O}$ data processing rates, we use three data security block (DSB) modules in the SEU/SDU design such that we can achieve the data throughput rate of $1 \mathrm{pixel} / \mathrm{cycle}$. This arrangement results in the pipeline operations on the DSB modules in the SEU/SDU.

Table 5 shows the scheduling of the DSB operations in the proposed design under pipelining and nonpipelining organization. In the pipelining organization (as the proposed design in Figure 5), we see that three DSBs perform the operations of loading/outputting, L/R rotation, and U/D rotation repeatedly so that the data throughput rate is equal to 1 Byte/cycle. The maximum throughput rate amounts to $117 \mathrm{Mbyte} / \mathrm{s}$ with the cycle time of 8.55 nanoseconds. On the other hand, if we only use one DSB instead of three in the SEU/SDU design, it is said to be in the nonpipelining orga- nization. In this case, we can trade off the hardware cost and the data throughput rate by removing two DSBs and lowering down the data throughput rate to $39 \mathrm{Mbyte} / \mathrm{s}$ in maximum. It leaves a freedom for users to select the pipelining or nonpipelining organizations when using the proposed design in different multimedia applications.

Besides, as we show in Figure 6, the control of the PEs is accomplished through the $\operatorname{ctrl}[4: 0]$ signal. The $\operatorname{ctrl}[4: 0]$ signal is composed of five control lines used in the PEs. For illustrating how to control the operations of PEs through $\operatorname{ctrl}[4: 0]$, we also show an example of assigning $\operatorname{ctrl}[4: 0]$ in Figure 6 when the PEs are operated in different operation modes like loading/outputting, L/R rotation, and U/D rotation. In case that the data is loaded into the $2 \mathrm{D}$ array in DSB, it is loaded through the ports, Ext_in and out, directly without using the four bidirectional data ports $\mathrm{Li}, \mathrm{Ri}, \mathrm{Ui}$, and Di. When the $\mathrm{L} / \mathrm{R}$ rotation and $\mathrm{U} / \mathrm{D}$ rotation operations are performed, the data is shifted through the four bidirectional ports under the control of $\operatorname{ctrl}[4: 0]$ as illustrated in Figure 6.

Figure 7 shows the architecture of the CBSG for the 1D logistic map. The parameters of $x(0)$ and $\mu$ are downloaded into the registers resided in the CBSG sequentially. Then, the CBSG performs the state evolution according to the behavior of the adopted 1D logistic map shown in (10). In order to minimize the hardware cost, only one multiplier is used for the state evolution. Each new state $x(n+1)$ is computed serially in two cycles according to the control signal shown in Table 6. At odd cycles, $\mu$ and $x(n)$ are multiplied. At even cycles, $\mu x(n)$ and $(1-x(n))$ are multiplied. That is, the CBSG generates 17 bits in the chaotic binary sequence every two cycles. Since the computation time for generating the chaotic binary sequence is much longer than that needed in the PEs, we use the design concept of multiple clock sources in the proposed design. That means we use a slower clock source in the CBSG design by dividing the original clock source by a factor. The value of the dividing factor should be determined by considering the consumption time of the PEs, multiplier in the CBSG, as well as the data consumption rate of the chaotic binary sequence. Besides, we also have to consider the complexity of the clock-dividing circuits and the synchronization between the two clocks. Table 7 shows the timing information and the control data consumption rate of the SEU/SDU and CBSGs in the proposed design. According to the results shown in Table 7, we find that the minimum consumption time per cycle in the SEU/SDU is about 8.55 nanoseconds and that the SEU/SDU consumes 17 bits of the control signals within 68.4 nanoseconds (in 8 cycles). Besides, we find that the minimum consumption 


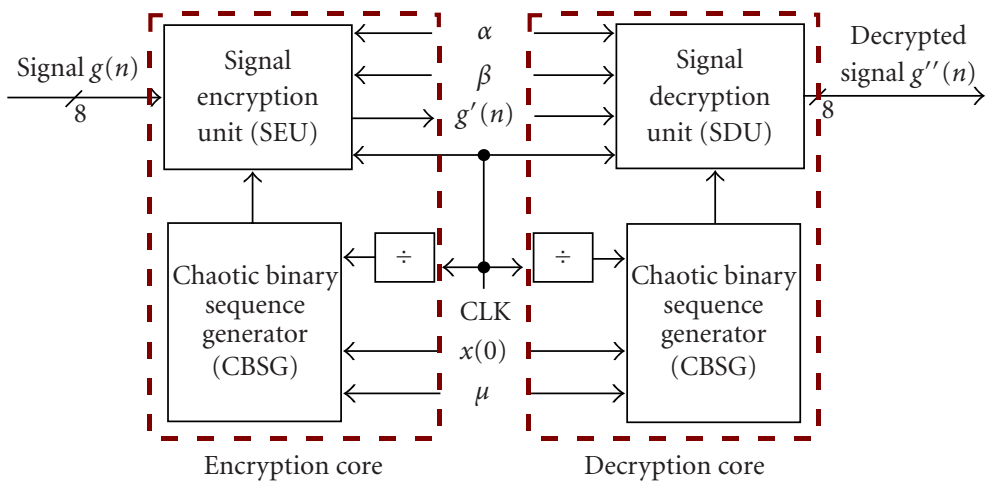

(a) Architecture.

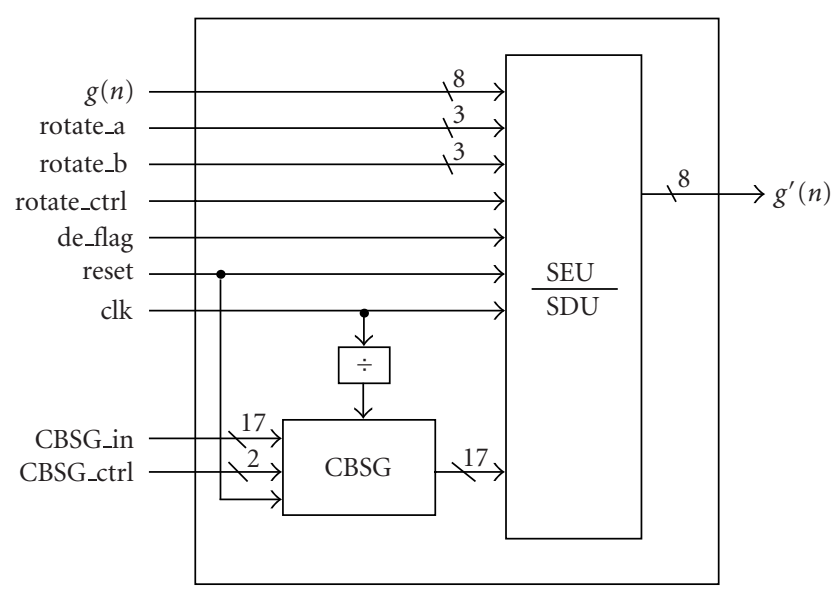

(b) Encryption/decryption core.

FIGURE 4: The architecture of the proposed signal security system.

per cycle in the CBSG is about 14.5 nanoseconds and that the CBSG generates 17 bits of the control signals within 29 nanoseconds (in 2 cycles for saving the hardware cost as we describe in Figure 7). Since the CBSG only uses $2 \mathrm{cy}-$ cles to generate 17 bits of the control signals that are consumed by the SEU/SDU in 8 cycles, the operating frequency of CBSG could be only $1 / 4$ of that used in the SEU/SDU. Therefore, we select the dividing factor to be 4 in dividing the clock of SEU/SDU before sending it to the CBSG. In this case, even if the operating frequency of the SEU/SDU achieves the maximum allowable $117 \mathrm{MHz}$, the operating frequency of that used in the CBSG should be $29.25 \mathrm{MHz}$ in maximum. Using this operating frequency in the CBSG will not have timing problems since we have 34.2 nanoseconds that is larger than the minimum time interval (i.e., 29 nanoseconds) to generate the 17 bits of the control signals by CBSG.

Figure 8 shows the clock-dividing circuit and the associated gate-level simulation results to illustrate the phenomenon of clock skew. From the gate-level simulation shown in Figure 8, we find that the clock skew period between the two clock sources used in SEU/SDU and CBSGs is about 1.14 nanosecond. Similar results are also found by examining the post-layout simulation. We have considered the problem of clock skew in deciding the clock dividing factor. Therefore, we can conclude that the clock skew problem of the two clock sources used in SEU/SDU and CBSGs does not have synchronization problems influencing the timing performance of the proposed design.

To verify the architecture of the proposed signal security system, we perform register transfer level (RTL) modeling of the proposed architecture by using Verilog HDL. In addition, we have performed the logic synthesis and the critical path analysis on the proposed architecture by using Synopsys logic synthesis tool DC based on a $0.35 \mu \mathrm{m}$ CMOS technology. Finally, we have implemented the proposed signal security system created by Avanti layout tools (Apollo). Table 8 shows the physical implementation results of the proposed signal security system. We found that the critical path of the proposed design is about 8.55 nanoseconds that achieves $117 \mathrm{MHz}$ data throughput rate. This processing speed can support up to 117 Mbytes/s in data encrypting and decrypting, which is fast enough to meet the real-time processing requirements of many digital image and 


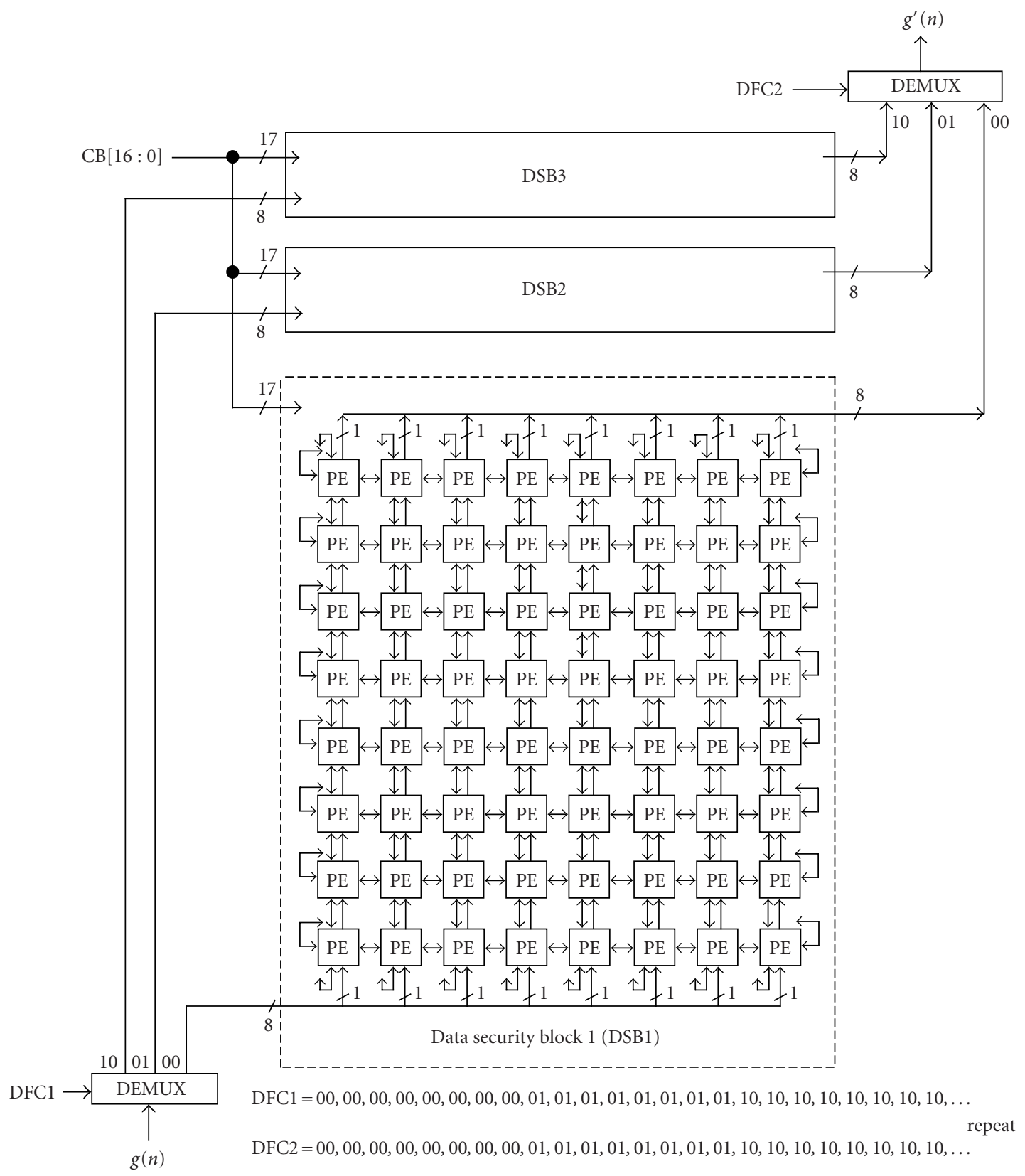

FIGURE 5: The architecture of the SEU/SDU in the proposed signal security system design.

video applications. Figure 9 shows the layout view according to a $0.35 \mu \mathrm{m}$ CMOS technology. The chip area of the proposed system is $3584443.28 \mu \mathrm{m}^{2}$.

\section{PERFORMANCE EVALUATION AND COMPARISONS}

This section provides the performance evaluation of the proposed design with other existing designs $[20,21,22,23,24$, 25]. In order to eliminate the factor of different fabrication technologies, we define an index of normalized area (denoted by NArea), which is the silicon area normalized to a $0.35 \mu \mathrm{m}$ technology as shown as follows:

$$
\text { NArea }=\frac{\text { Area }}{(\text { Technology/0.35 })^{2}} .
$$

Besides, we also define an index of data rate per area (DRPA), that is, Data rate/NArea, as shown in (21), to reflect the efficiency of the hardware design for data encrypter and decrypter. It is shown as follows:

$$
\text { DRPA }=\frac{\text { Data }- \text { rate }}{\text { NArea }} \quad \mathrm{Mbps} / \mathrm{mm}^{2} .
$$



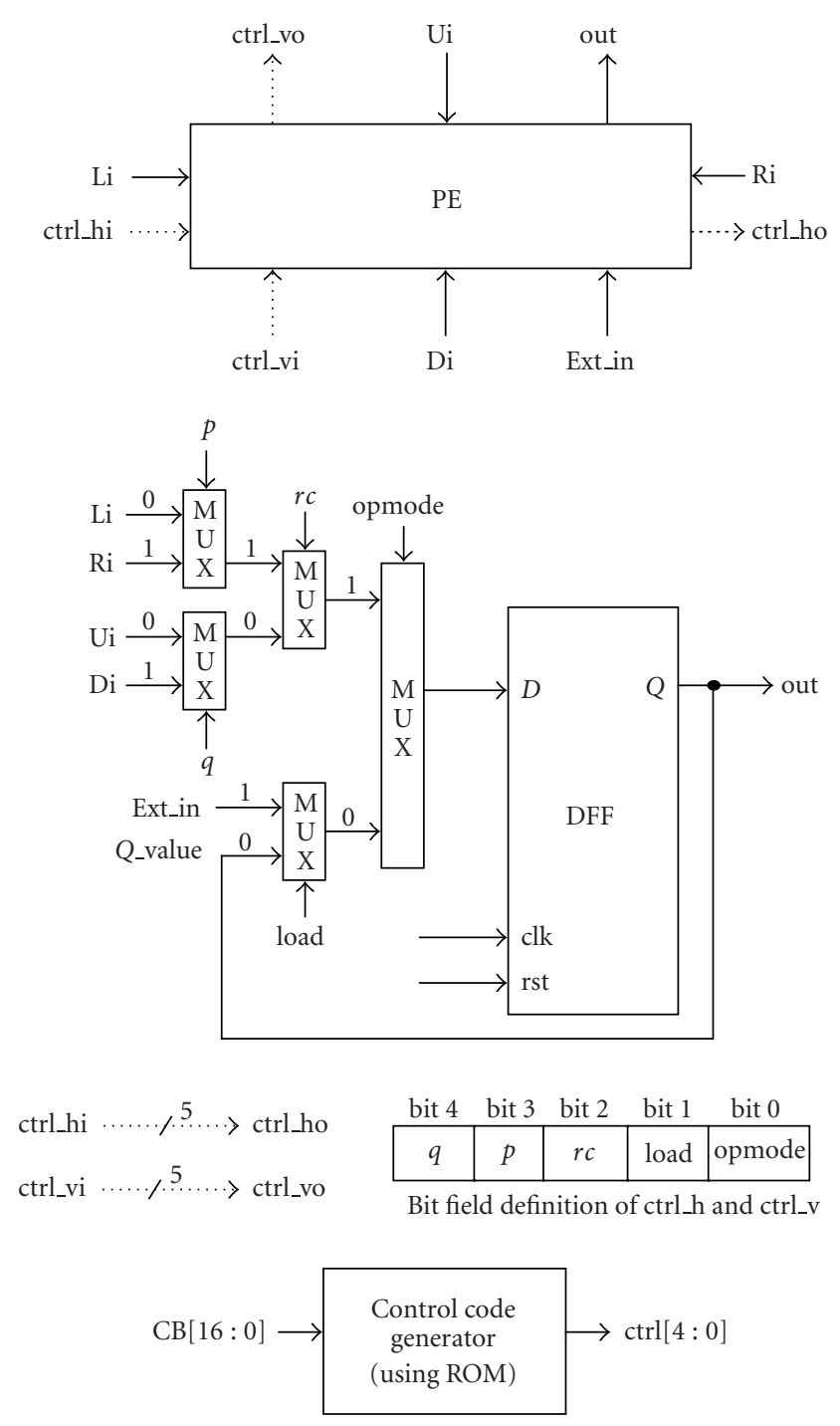

$\operatorname{ctrl}[4: 0]$ code assignment

\begin{tabular}{|c|c|}
\hline Operations & $\operatorname{ctrl}[4: 0]$ \\
\hline Loading/outputing & 00010 \\
\hline L/R rotation & $\begin{array}{c}0 p 101 \text { in the first } r \text { cycles } \\
0 p 100 \text { in the remaining cycles }\end{array}$ \\
\hline U/D rotation & $\begin{array}{c}q 001 \text { in the first } s \text { cycles } \\
q 0000 \text { in the remaining cycles }\end{array}$ \\
\hline
\end{tabular}

FIgUre 6: The architecture of the PEs used in the DSB in the proposed SEU/SDU.

Based on the above two indices, we have summarized the comparison results of the proposed design with the existing ones $[20,21,22,23,24,25]$ in Table 9. From Table 9, we find that the proposed design is better than the design in [20] in providing higher data processing rate at lower hardware cost weighted using gate count based on a $0.35 \mu \mathrm{m}$ CMOS technology. Also, the proposed design achieves higher data-processing rate at lower hardware cost in gate count as compared with the design in [25]. As for the comparison to the other popular data encryption/decryption approaches $[21,22,23,24,25]$, we use the normalized index of DRPA defined in (21) in the evaluation of the efficiency in these approaches. We find that the proposed design is better than the existing designs in $[21,22,23,24]$ in possessing higher DRPA. That means that the proposed design can provide higher efficiency considering the data processing rate and required hardware cost. Though the above comparison cannot provide the absolute comparison in every aspect (including 


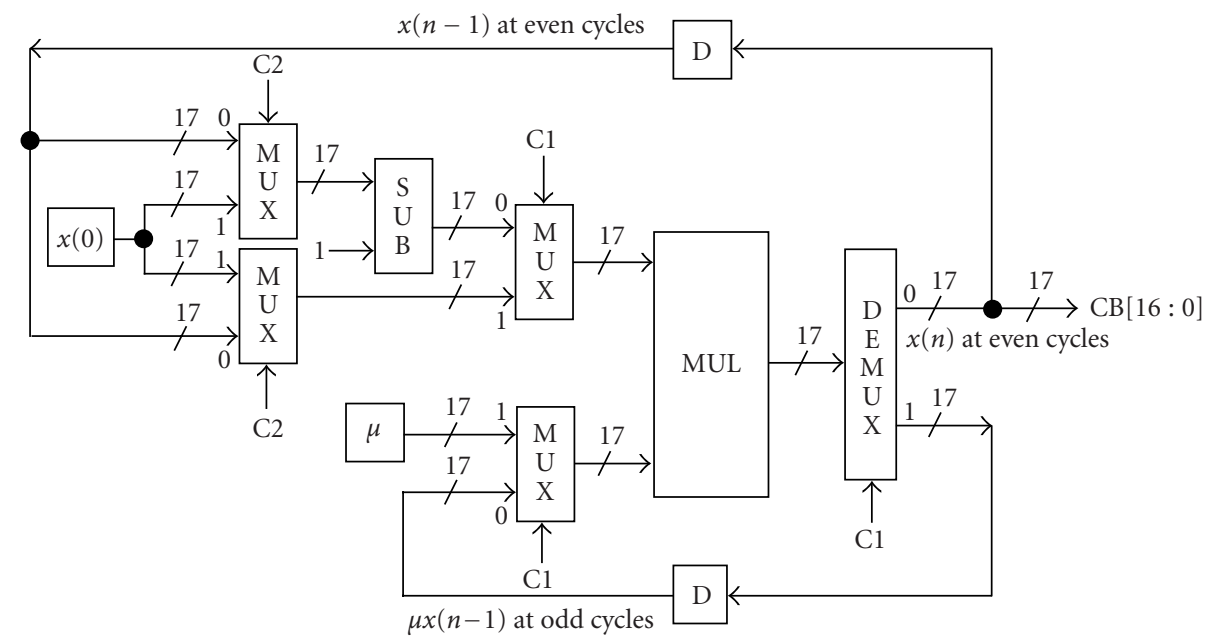

FIGURE 7: The architecture of the CBSG used in the proposed signal security system.

TABLE 5: The scheduling of DSB operations in the proposed design, where Dn denotes the $n$th set (with 8 bytes in a set) of data.

(a) Pipelining organization (using three DSBs as proposed in Figure 5).

\begin{tabular}{r|cccc}
\hline \multirow{2}{*}{ Cycle } & \multicolumn{4}{|c}{ DSB modules } \\
\cline { 2 - 5 } $0 \sim 7$ & DSB1 & DSB2 & DSB3 & Data output \\
\hline $8 \sim 15$ & L/R rotation on D1 & Load D2 & - & - \\
$16 \sim 23$ & U/D rotation on D1 & L/R rotation on D2 & Load D3 & - \\
$24 \sim 31$ & Load D4/output D1 & U/D rotation on D2 & L/R rotation on D3 & D1 \\
$32 \sim 39$ & L/R rotation on D4 & Load D5/output D2 & U/D rotation on D3 & D2 \\
$40 \sim 47$ & U/D rotation on D4 & L/R rotation on D5 & Load D6/output D3 & D3 \\
$48 \sim 55$ & Load D7/output D4 & U/D rotation on D5 & L/R rotation on D6 & D4 \\
$56 \sim 63$ & L/R rotation on D7 & Load D8/output D5 & U/D rotation on D6 & D5 \\
$64 \sim 71$ & U/D rotation on D7 & L/R rotation on D8 & Load D9/output D6 & D6 \\
$72 \sim 79$ & - & U/D rotation on D8 & L/R rotation on D9 & D7 \\
$80 \sim 87$ & - & - & U/D rotation on D9 & D8 \\
\hline
\end{tabular}

(b) Nonpipelining organization (using only one DSB).

\begin{tabular}{c|cc}
\hline \multirow{2}{*}{ Cycle } & \multicolumn{2}{|c}{ DSB modules } \\
\cline { 2 - 3 } & DSB & Data output \\
\hline $0 \sim 7$ & Load D1 & - \\
$8 \sim 15$ & L/R rotation on D1 & - \\
$16 \sim 23$ & U/D rotation on D1 & - \\
$24 \sim 31$ & Load D2/output D1 & D1 \\
$32 \sim 39$ & L/R rotation on D2 & - \\
$40 \sim 47$ & U/D rotation on D2 & - \\
$48 \sim 55$ & Load D3/output D2 & D2 \\
$56 \sim 63$ & L/R rotation on D3 & - \\
$64 \sim 71$ & U/D rotation on D3 & - \\
$72 \sim 79$ & Load D4/output D3 & D3 \\
$80 \sim 87$ & - & - \\
\hline
\end{tabular}


TABLE 6: The assignments of the control signals C1 and C2 in the CBSG.

\begin{tabular}{c|ccccccccccc}
\hline Time & 1 & 2 & 3 & 4 & 5 & 6 & 7 & 8 & 9 & 10 \\
\hline $\mathrm{C} 1$ & 1 & 0 & 1 & 0 & 1 & 0 & 1 & 0 & 1 & 0 \\
$\mathrm{C} 2$ & 1 & 1 & 0 & 0 & 0 & 0 & 0 & 0 & 0 & 0 \\
Output & - & $\mathrm{CB}[16: 0]$ & - & $\mathrm{CB}[16: 0]$ & - & $\mathrm{CB}[16: 0]$ & - & $\mathrm{CB}[16: 0]$ & - & $\mathrm{CB}[16: 0]$ & $\cdots$ \\
\hline
\end{tabular}

TABLE 7: The timing information and the consumption rate of the control signals in the SEU/SDU and CBSGs.

\begin{tabular}{l|cllll}
\hline & $\begin{array}{l}\text { Minimum consumption time } \\
\text { with clock skew per cycle }(\mathrm{ns})\end{array}$ & $\begin{array}{l}\text { Consumption/generation } \\
\text { rate in control signals }\end{array}$ & $\begin{array}{l}\text { Clock dividing factor } \\
\left(F_{\text {SEU/SDU }} / F_{\text {CBSG }}\right)\end{array}$ & $\begin{array}{l}\text { Maximum clock } \\
\text { frequency }(\mathrm{MHz})\end{array}$ & $\begin{array}{l}\text { Meeting critical } \\
\text { path constraint }\end{array}$ \\
\hline SEU/SDU & 8.55 & 17 bits/68.4 ns (in 8 cycles $)$ & $4(=8$ cycles/2 cycles $)$ & 117 & Yes \\
CBSGs & $14.5(=13.36+1.14)$ & 17 bits/29 ns (in 2 cycles $)$ & $4(=8$ cycles/2 cycles $)$ & $29.25(=117 / 4)$ & Yes \\
\hline
\end{tabular}

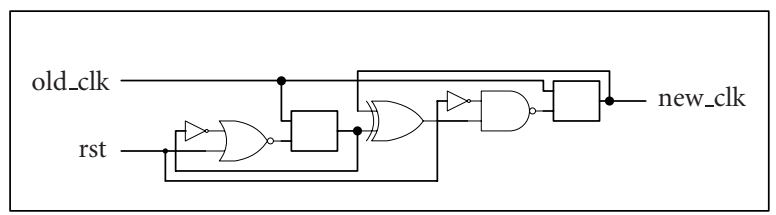

(a) Clock dividing circuit optimized by Synopsys design compiler.

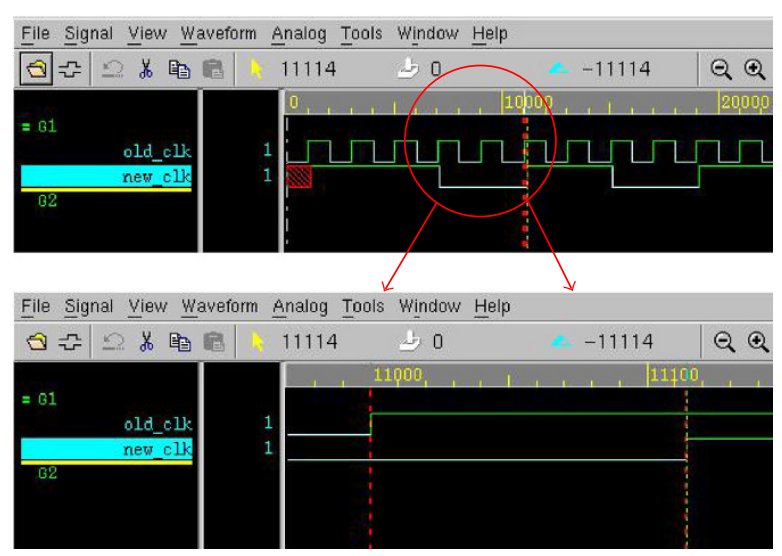

(b) Gate-level simulation regarding clock skew (clock skew is about 1.14 nanosecond).

FIGURE 8: Clock dividing circuit and the associated gate-level simulation results regarding clocks $\left(\mathrm{CLK}_{\mathrm{SEU} / \mathrm{SDU}}=\right.$ old_clk and $\mathrm{CLK}_{\mathrm{CBSG}}=$ new_clk) in the proposed design.

the efficiency as well as other factors like security and so on) between the proposed design and others, it can reflect the efficiency of the proposed design under the situation that the security of the proposed design and the existing ones $[20,21,22,23,24,25]$ is good enough for the applications.

When the proposed TDCEA design is applied to the data protection in the multimedia data transmission, we may face a problem that the encrypted data is lost or corrupted by noise. In the proposed design, it uses 8 -byte data as a unit
TABle 8: The details of the physical implementation on the proposed signal security system.

\begin{tabular}{ll}
\hline Item & Result \\
\hline Technology & COMPASS $0.35 \mu \mathrm{m}$ CMOS \\
Power supply & $3.3 \mathrm{~V}$ \\
Area in gate count & 12721 gates \\
Critical path delay & $8.55 \mathrm{~ns}$ \\
Chip size & $3584443.28 \mu \mathrm{m}^{2}\left(\sim 3.59 \mathrm{~mm}^{2}\right)$ \\
Power consumption & $20 \mathrm{~mW} 50 \mathrm{MHz}$ \\
\hline
\end{tabular}

in the encrypting or decrypting processes. Different units of 8-byte data are encrypted or decrypted independently except for using the neighboring bit-stream data as the control signals. If there is any loss or data corruption in the encrypted data when transmitted in the network, it would cause data errors in the around 8-byte data that contains the lost one. And the error will not propagate if we also flush the associated control signals with the corrupted data. Since the proposed design is targeted at the applications of multimedia data transmission, the errors caused by the interference from network could be recovered by exploiting the well-developed multimedia error concealment approaches proposed in the literature.

\section{CONCLUSION}

In this paper, we have proposed a new signal security system with its VLSI architecture designed, verified, and physically realized by using Verilog HDL and Synopsys logic synthesis tool (Design Compiler), and Avanti layout tools (Apollo) based on a $0.35 \mu \mathrm{m}$ CMOS technology. The features of the proposed signal security system include $O(N)$ computational complexity, high security, and low hardware cost. The simulation results have indicated that (1) the encryption results are completely chaotic by the sense of sight or by the high fractal dimension, and (2) the encryption results are very sensitive to the parameter fluctuation. In addition, we have implemented the proposed design in silicon that possesses 


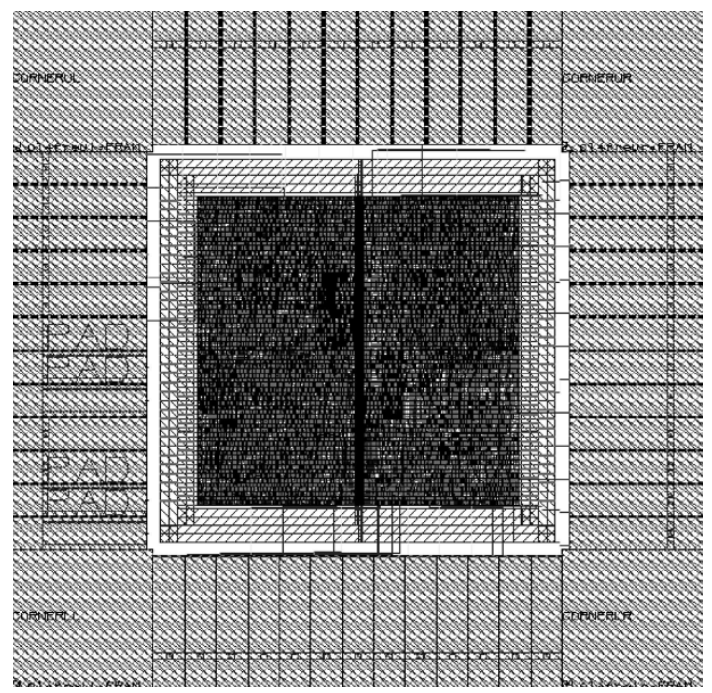

FIGURE 9: The layout view of the proposed signal security system implemented using $0.35 \mu \mathrm{m}$ CMOS technology.

TABle 9: Comparison of the proposed design with the existing designs [20, 21, 22, 23, 24, 25].

\begin{tabular}{|c|c|c|c|c|c|c|c|c|}
\hline Design & $\begin{array}{l}\text { Technology } \\
(\mu \mathrm{m})\end{array}$ & $\begin{array}{l}\text { Area } \\
\left(\mathrm{mm}^{2}\right)\end{array}$ & $\begin{array}{l}\text { Gate } \\
\text { count }\end{array}$ & $\begin{array}{l}\text { Data rate } \\
\text { (Mbits/s) }\end{array}$ & $\begin{array}{l}\text { Normalized area } \\
\text { (NArea) }\left(\mathrm{mm}^{2}\right)\end{array}$ & $\begin{array}{l}\text { Data rate/NArea } \\
(\mathrm{DRPA})\left(\mathrm{Mbps} / \mathrm{mm}^{2}\right)\end{array}$ & $\begin{array}{l}\text { Encryption } \\
\text { algorithm }\end{array}$ & $\begin{array}{l}\text { Cipher block } \\
\text { length (bit) }\end{array}$ \\
\hline Design [20] & 0.35 & - & 55345 & 395 & - & - & FEA-M & 64 \\
\hline Design [21] & 0.7 & 29 & - & 251.8 & 7.25 & 34.73 & SAFER K-128 & 64 \\
\hline Design [22] & 0.35 & 13.69 & 51400 & 600 & 13.69 & 43.82 & AES & 128 \\
\hline Design [23] & 1.2 & 107.8 & - & 177.8 & 9.17 & 19.39 & IDEA & 64 \\
\hline Design [24] & 0.8 & 11.7 & - & 355 & 2.24 & 158.53 & IDEA & 64 \\
\hline Design [25] & 0.25 & - & 31957 & 609 & - & - & AES & 128 \\
\hline Proposed & 0.35 & 3.59 & 12721 & 856 & 3.59 & 238.44 & TDCEA & 64 \\
\hline
\end{tabular}

the data processing rate up to $117 \mathrm{Mbytes} / \mathrm{s}$ at the cost of silicon area of $3.59 \mathrm{~mm}^{2}$. The comparison results of the proposed design with other existing designs show that the proposed design possesses better performance in terms of the evaluation index of DRPA, which shows the efficiency of the proposed design. Finally, it is believed that many real-time digital signal processing systems can benefit from the integration with the proposed signal security system for providing high data security in data storage and transmission.

\section{REFERENCES}

[1] S. Moni and R. L. Kashyap, "Image communication over a distributed multimedia system," IEEE Journal on Selected Areas in Communications, vol. 14, no. 7, pp. 1472-1483, 1996.

[2] Y. Yamamoto, F. Inumaru, S. D. Akers, and K. I. Nishimura, "Transmission performance of $64 \mathrm{Kbps}$ switched digital international ISDN connections," IEEE Trans. Communications, vol. 42, no. 12, pp. 3215-3220, 1994.

[3] W. Diffie and M. E. Hellman, "Privacy and authentication: an introduction to cryptography," Proceedings of the IEEE, vol. 67, no. 3, pp. 397-427, 1979.
[4] M. E. Smid and D. K. Branstad, "The data encryption standard: past and future," Proceedings of the IEEE, vol. 76, no. 5, pp. 550-559, 1988.

[5] J.-C. Yen and J.-I. Guo, "An efficient hierarchical chaotic image encryption algorithm and its VLSI realization," IEE Proceedings - Vision, Image and Signal Processing, vol. 147, no. 2, pp. 167-175, 2000.

[6] N. Bourbakis and C. Alexopoulos, "Picture data encryption using SCAN patterns," Pattern Recognition, vol. 25, no. 6, pp. 567-581, 1992.

[7] P. Refregier and B. Javidi, "Optical-image encryption based on input plane and Fourier plane random encoding," Optics Letters, vol. 20, no. 7, pp. 767-769, 1995.

[8] J.-C. Yen and J.-I. Guo, "A chaotic neural network for signal encryption/decryption and its VLSI architecture," in Proc. 10th VLSI Design/CAD Symposium, pp. 319-322, Nan-Tou, Taiwan, August 1999.

[9] T. J. Chuang and J. C. Lin, "A new multiresolution approach to still image encryption," Pattern recognition and Image Analysis, vol. 9, no. 3, pp. 431-436, 1999.

[10] C. J. Kuo and M. S. Chen, "A new signal encryption technique and its attack study," in Proc. IEEE International Carnahan Conference On Security Technology, pp. 149-153, Taipei, Taiwan, October 1991. 
[11] C. T. Hsu and J. L. Wu, "Hidden digital watermarks in images," IEEE Trans. Image Processing, vol. 8, no. 1, pp. 58-68, 1999.

[12] B. M. Macq and J.-J. Quisquater, "Cryptology for digital TV broadcasting," Proceedings of the IEEE, vol. 83, no. 6, pp. 944957, 1995.

[13] Z. Xiong and Y. Q. Zhang, "Multiresolution watermarking for images and video," IEEE Trans. Circuits and Systems for Video Technology, vol. 9, no. 4, pp. 545-550, 1999.

[14] C. W. Wu and N. F. Rulkov, "Studying chaos via 1-D maps-a tutorial," IEEE Trans. on Circuits and Systems I: Fundamental Theory and Applications, vol. 40, no. 10, pp. 707-721, 1993.

[15] H. G. Schuster, Deterministic Chaos: An Introduction, PhysikVerlag, Weinheim, Germany, 1984.

[16] T. S. Parker and L. O. Chua, "Chaos: a tutorial for engineers," Proceedings of the IEEE, vol. 75, no. 8, pp. 982-1008, 1987.

[17] E. Biham, "Cryptanalysis of the chaotic-map cryptosystem suggested at Eurocrypt '91," in Advances in CryptologyEurocrypt '91: Proc. Workshop on the Theory and Application of Cryptographic Techniques, pp. 532-534, Springer-Verlag, Berlin, April 1991.

[18] A. E. Jacquin, "Fractal image coding: A review," Proceedings of the IEEE, vol. 81, no. 10, pp. 1451-1465, 1993.

[19] C. C. Chen, J. S. Daponte, and M. D. Fox, "Fractal feature analysis and classification in medical imaging," IEEE Trans. on Medical Imaging, vol. 8, no. 2, pp. 133-142, 1989.

[20] X. Yi, C. H. Tan, C. K. Siew, and M. R. Syed, "Fast encryption for multimedia," IEEE Transactions on Consumer Electronics, vol. 47, no. 1, pp. 101-107, 2001.

[21] A. Schubert, V. Meyer, and W. Anheier, "Reusable cryptographic VLSI core based on the SAFER K-128 algorithm with 251.8 Mbit/s throughput," in Proc. IEEE Workshop on Signal Processing Systems, pp. 437-446, Cambridge, Mass, USA, October 1998.

[22] Y. Mitsuyama, Z. Andales, T. Onoye, and I. Shirakawa, "VLSI implementation of high performance burst mode for 128-bit block ciphers," in Proc. IEEE Int. Symp. Circuits and Systems, vol. 2, pp. 344-347, Sydney, Australia, May 2001.

[23] A. Curiger, H. Bonnenberg, R. Zimmermann, N. Felber, H. Kaeslin, and W. Fichtner, "VINCI: VLSI implementation of the new secret-key block cipher IDEA," in Proc. IEEE Custom Integrated Circuits Conference, pp. 15.5.1-15.5.4, San Diego, Calif, USA, May 1993.

[24] S. Wolter, H. Matz, A. Schubert, and R. Laur, "On the VLSI implementation of the internal data encryption algorithm IDEA," in Proc. IEEE Int. Symp. Circuits and Systems, vol. 1, pp. 397-400, Seattle, Wash, USA, 1995.

[25] C.-C. Lu and S.-Y. Tseng, "Integrated design of AES (Advanced Encryption Standard) encrypter and decrypter," in Proc. IEEE 13th International Conference on ApplicationSpecific Systems, Architectures, and Processors, pp. 277-285, San Jose, Calif, USA, July 2002.

Hun-Chen Chen was born in Taipei, Taiwan, in 1961. He received the B.S. and M.S. degrees, all in electronics engineering, from the National Taiwan Technology University, Taipei, Taiwan, and National Chiao Tung University, Hsinchu, Taiwan, in 1990 and 1998, respectively. He is currently an Instructor in the Department of Electronics Engineering, National United University, Miaoli, Taiwan. His research interests include neural network application, digital signal processing, and VLSI architecture design.

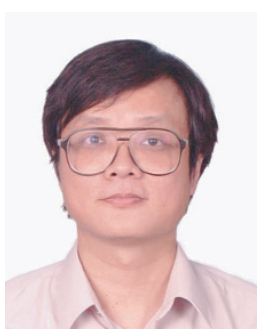

Jiun-In Guo was born in Kaohsiung, Taiwan, in 1966. He received the B.S. and Ph.D. degrees in electronics engineering from $\mathrm{Na}$ tional Chiao Tung University, Hsinchu, Taiwan, in 1989 and 1993, respectively. He is currently a Professor in the Department of Computer Science and Information Engineering, National Chung-Cheng University, Chiayi, Taiwan, where he was an Associate Professor from 2001 to 2003. He was an As-

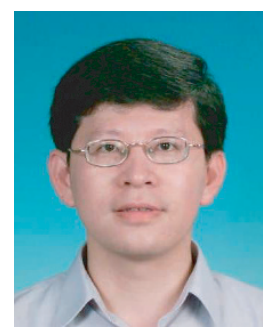
sociate Professor in the Department of Electronics Engineering, National Lien-Ho Institute of Technology from 1994 to 2001 and Director of the same department from 1996 to 1999. His research interests include image, speech, and digital signal processing, VLSI architecture design, VLSI implementation, digital IP design, and SOC design.

Lin-Chieh Huang was born in Kaohsiung, Taiwan, in 1979. He received the B.S. degree from the Department of Computer Science and Information Engineering, $\mathrm{Na}$ tional Chi Nan University, Nan-tou, Taiwan, in 2002. He is currently working on his M.S. degree in the Department of Computer Science and Information Engineering, National Chung Cheng University. His research interests include video processing algorithm, VLSI architecture design, digital IP design, SOC hardware design, multimedia ASIC/IP/SOC design, image signal processing, and computer vision.

Jui-Cheng Yen was born in Tainan, Taiwan, in 1963. He received the B.S. and Ph.D. degrees in electrical engineering from $\mathrm{Na}-$ tional Tsing Hua University, Hsinchu, Taiwan, in 1987 and 1992, respectively. He is currently a Professor in the Department of Electronics Engineering, National United University, Miaoli, Taiwan. He was Director of the Department of Electronics Engineering, National Lien-Ho Institute of Technol-

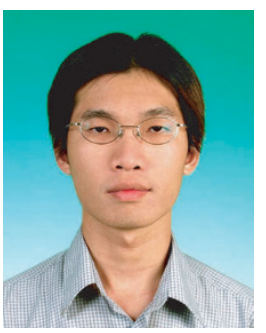
ogy, Miaoli, Taiwan in 2002. His research interests include image, speech, and digital signal processing, VLSI architecture design, and artificial neural network.

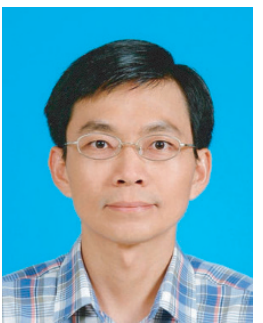

